\title{
Acesso à detecção precoce do câncer de mama no Sistema Único de Saúde: uma análise a partir dos dados do Sistema de Informações em Saúde
}

\author{
Access to early breast cancer diagnosis in the \\ Brazilian Unified National Health System: \\ an analysis of data from the Health \\ Information System
}

\section{El acceso a la detección temprana del cáncer de mama en el Sistema de Salud de Brasil: un análisis de datos del Sistema de \\ Información de Salud}

\begin{abstract}
The recent reduction in breast cancer mortality in high-income countries resulted from improvements in early detection and treatment. Breast cancer is the most common cancer in Brazilian women. Since 2004, the government has recommended annual clinical breast examination for women aged $\geq 40$ years and biannual mammograms for those aged 50-69. This article investigates the degree of implementation of these guidelines using data from the Brazilian Unified National Health System for 2010 according to major geographic region and age group. The findings showed low national mammogram coverage in the target population (32\% in the 50-59-year group; 25\% from 60 to 69 years). The percentage of women with abnormal radiological findings who underwent biopsy was also low (27\% for 50-59 years; $63 \%$ for $60-69$ years). The number of breast cancer surgeries exceeded the number of cases detected by mammography but was well below the estimated number of incident breast cancer cases in 2010. There are striking regional inequalities in access to early detection and surgery, being the lowest access in the North Region and the highest in the South Region.
\end{abstract}

Breast Neoplasms; Early Detection of Cancer; Women's Health; Mass Screening
Gulnar Azevedo e Silva 1

Maria Teresa Bustamante-Teixeira 2

Estela M. L. Aquino 3

Jeane Glaucia Tomazelli 4

Isabel dos-Santos-Silva ${ }^{5}$

\section{Resumo}

A redução recente na mortalidade por câncer de mama em países de alta renda é atribuída à detecção precoce e melhorias no tratamento. $O$ câncer de mama é o tipo mais frequente de câncer feminino no Brasil, e, desde 2004, o governo recomenda o exame clínico anual das mamas para mulheres a partir dos 40 anos e rastreio mamográfico bienal entre 50 e 69 anos. Este artigo investiga o nível de implementação dessas recomendações usando os dados dos sistemas de informações do SUS de 2010 por macrorregião e grupo etário. Evidenciou-se uma cobertura baixa de mamografia entre a população alvo (32\%: 50-59 anos; 25\%: 60-69 anos). A proporção de mulheres com achados radiológicos anormais submetidas à biópsia também foi baixa (27\%: 50-59 anos; 63\%: 60-69 anos). O número de cirurgias para câncer de mama foi maior do que o número de casos detectáveis pela mamografia, mas muito inferior ao número estimado de casos incidentes para 2010. Existem marcadas desigualdades regionais no acesso à detecção precoce e à cirurgia, sendo o acesso mais baixo na Região Norte e mais alto na Região Sul.

Neoplasias de Mama; Detecção Precoce de Câncer; Saúde da Mulher; Programas de Rastreamento 


\section{Introdução}

A ocorrência do câncer de mama varia entre países segundo o grau de desenvolvimento socioeconômico, sendo as taxas nos países de alta renda muito superiores às de média e baixa renda 1,2,3,4 No entanto, não existe um diferencial claro na mortalidade pela doença porque esses últimos ainda têm índices de sobrevida por câncer de mama muito baixos 1,4. A melhoria na sobrevida das pacientes em países desenvolvidos nas últimas décadas foi decorrente do aumento do alerta sobre a doença, da detecção precoce e do aprimoramento do tratamento 4 .

O rastreamento populacional do câncer de mama foi introduzido em alguns países europeus, nos Estados Unidos e no Canadá, no final dos anos de 1980, após ensaios clínicos randomizados terem mostrado que o rastreamento mamográfico era responsável pela diminuição de $20 \%$ a $30 \%$ na mortalidade por essa neoplasia 5,6,7. Entretanto, existem controvérsias em relação aos benefícios e aos efeitos indesejados de tais programas e à sua real contribuição no declínio da mortalidade pela doença. Revisão recente por um painel independente confirmou uma redução de $20 \%$ na mortalidade por câncer de mama em participantes de um programa de rastreamento de 20 anos de seguimento; porém, essa redução foi acompanhada de efeitos nocivos como sobrediagnóstico de casos, resultados falsos negativos e falsos positivos 8 .

Alguns países da América Latina introduziram ações de rastreamento para o câncer de mama na última década, mas, até o momento, não foi implementado, na região, um programa organizado de base populacional 9 . No Brasil, onde o câncer de mama é o principal tipo de neoplasia maligna que afeta as mulheres, o Ministério da Saúde preconiza, desde 2004, o exame clínico anual para mulheres assintomáticas a partir dos 40 anos de idade e a mamografia bienal para as mulheres entre $50 \mathrm{e}$ 69 anos - com recomendações mais intensas para as que pertencem a grupos de alto risco 10. Após quase uma década, não foram localizados estudos que avaliem, em âmbito nacional, o grau de implantação dessas recomendações e os resultados obtidos. Tampouco foi avaliada, até o momento, a capacidade do Sistema Único de Saúde (SUS) de produzir serviços para cumprir seus propósitos de controle do câncer de mama no país e, em última instância, atender às necessidades de saúde das mulheres brasileiras.

Em 2009, foi implantado o Sistema de Informação para o Controle do Câncer de Mama (SISMAMA) 11, para padronização da coleta de dados sobre o rastreamento, o diagnóstico e o tratamento do câncer da mama em todo o país e permitir a avaliação das ações de controle da doença 12,13.
Avaliações sobre o rastreamento do câncer de mama com base no SISMAMA, publicadas até o momento, limitaram-se a descrever as características principais do sistema 12 e a estimar sua cobertura e qualidade com abrangência regional 13 .

Pretende-se, neste artigo, avaliar a cobertura do rastreamento do câncer de mama na população alvo e o seguimento de lesões mamográficas sugestivas de malignidade e examinar se o número de achados mamográficos anormais e o volume de cirurgias realizadas são apropriados, dadas as estimativas de incidência do câncer de mama na população dependente do SUS para o Brasil e grandes regiões, segundo grupos etários.

\section{Métodos}

Foi realizado estudo descritivo com base em dados secundários dos Sistemas de Informações de Saúde do SUS referentes ao ano de 2010.

\section{Fonte de dados}

Foram utilizados os dados do Sistema de Informações Ambulatoriais (SIA), do SISMAMA, do Sistema de Informações Hospitalares (SIH) e do Sistema de Informações sobre Mortalidade (SIM), referentes a mulheres com idade igual ou superior a 40 anos em 2010, para o Brasil e grandes regiões (Departamento de Informática do SUS. Informações em Saúde. http://www2.datasus.gov.br/DATASUS/in dex.php?area=02). Os dados da população para o cálculo de denominadores foram obtidos do censo decenal de 2010 (Instituto Brasileiro de Geografia e Estatística. Sinopse do Censo Demográfico 2010. http://www.ibge.gov.br).

\section{Fluxo de informação}

Os procedimentos relativos a rastreamento, diagnóstico e tratamento cirúrgico para o câncer de mama são registrados em diferentes Sistemas de Informações do SUS (DATASUS. Informações em Saúde. http://www2.datasus.gov.br/DATASUS/in dex.php?area=02).

O SIA e o SIH foram implantados na década de 1990, com finalidade administrativa e contábil, sendo baseados em procedimentos e não em indivíduos. No SIA, são registrados aqueles realizados em âmbito ambulatorial, por meio do Boletim de Produção Ambulatorial. No SIH, são registradas as Autorizações de Internação Hospitalar (AIH) referentes a intervenções cirúrgicas para o diagnóstico e o tratamento do câncer de mama mediante solicitação com laudo médico (DATASUS. Informações em Saúde. http://www2.datasus.gov. $\mathrm{br} / \mathrm{DATASUS} /$ index.php?area=02). 
No SISMAMA, subsistema do SIA instalado nos serviços públicos ou nos prestadores contratados pelo SUS e nas Coordenações de Saúde da Mulher das secretarias estaduais e municipais de saúde, são registrados os dados de solicitação e resultados de exames de mamografia, citopatológicos e anatomopatológicos de mama (biópsia e peça cirúrgica) 12 .

Na unidade de saúde, o profissional, ao identificar uma usuária assintomática dentro da faixa etária preconizada pelo Ministério da Saúde 10, deve solicitar a "mamografia de rastreamento" em formulário padronizado e fazer o seu encaminhamento. Para mulheres sintomáticas de qualquer faixa etária, a solicitação deve ser feita com indicação clínica de "mamografia diagnóstica".

O laudo de mamografia padronizado com base no sistema de classificação Breast Imaging Reporting and Data System (BI-RADS) 14 é entregue à mulher, a qual deve se dirigir ao profissional que solicitou o exame para conduta apropriada. Por sua vez, o encaminhamento para fechar o diagnóstico apoia-se na interpretação da categoria BI-RADS (Categoria 0 - inconclusivo; Categoria 1 - sem achados; Categoria 2 - achados benignos; Categoria 3 - provavelmente benigno; Categoria 4 - suspeito de malignidade; Categoria 5 - altamente sugestivo de malignidade e Categoria 6 - biópsia prévia com malignidade comprovada).
As biópsias são realizadas em unidades secundárias de referência para patologias mamárias e em hospitais do SUS, e o diagnóstico histológico também deve ser registrado no SISMAMA. Quando há confirmação do diagnóstico, havendo indicação de tratamento cirúrgico, a mulher é encaminhada para hospitais especializados em cirurgia mamária, e o procedimento é registrado então no SIH (Tabela 1).

Para receber o pagamento, é necessário que o serviço que realizou qualquer um dos procedimentos gere um Boletim de Produção Ambulatorial no SIA. Nesse momento, as informações epidemiológicas devem ser enviadas para as secretarias de saúde para a consolidação, no SISMAMA, dos dados de cada estado.

As declarações de óbito em todo o país são registradas no SIM que hoje atinge uma cobertura superior a $90 \%$.

\section{Indicadores utilizados}

Foram construídos indicadores de ocorrência do câncer de mama (incidência e mortalidade) - que expressam as necessidades de saúde - e aqueles relativos aos procedimentos efetuados no rastreamento, diagnóstico e tratamento cirúrgico da doença - que traduzem o acesso efetivo ou uso dos serviços 15. As análises foram procedidas compa-

Tabela 1

Fluxo das mulheres para realização de mamografia, biópsia e cirurgia para câncer de mama segundo local de atendimento e registro em sistema de informação do SUS.

\begin{tabular}{|c|c|c|}
\hline Nível de atenção & Percurso da mulher & Sistema de informação \\
\hline $\begin{array}{l}\text { Unidade de saúde } \\
\text { (atenção básica) }\end{array}$ & Recebimento da solicitação de mamografia & \\
\hline $\begin{array}{l}\text { Serviço de mamografia } \\
\text { (atenção secundária) }\end{array}$ & Realização da mamografia e recebimento do resultado & SIA; SISMAMA \\
\hline $\begin{array}{l}\text { Unidade de saúde } \\
\text { (atenção básica) }\end{array}$ & $\begin{array}{l}\text { Retorno ao médico solicitante com o laudo da mamografia. Se o laudo da mamografia for } \\
\text { Categoria BI-RADS } 4 \text { ou 5, é feito o encaminhamento para unidade especializada }\end{array}$ & \\
\hline $\begin{array}{l}\text { Unidade especializada } \\
\text { (atenção secundária) }\end{array}$ & Recebimento da solicitação e realização da biópsia & \\
\hline $\begin{array}{l}\text { Laboratório (atenção } \\
\text { secundária) }\end{array}$ & Emissão do resultado da biópsia & SIA; SISMAMA \\
\hline $\begin{array}{l}\text { Unidade especializada } \\
\text { (atenção secundária) }\end{array}$ & $\begin{array}{c}\text { Se o laudo da biópsia refere doença benigna, é feita orientação médica } \\
\text { sobre a conduta terapêutica. Se o laudo da biópsia refere doença maligna, } \\
\text { é feito encaminhamento para a cirurgia }\end{array}$ & \\
\hline $\begin{array}{l}\text { CACON/UNACON } \\
\text { (atenção terciária) }\end{array}$ & Realização da cirurgia. Tratamento & $\mathrm{SIH}$ \\
\hline
\end{tabular}

BI-RADS: Breast Imaging Reporting and Data System; CACON: Centro de Alta Complexidade em Oncologia; SIA: Sistema de Informações Ambulatoriais; SIH: Sistema de Informações Hospitalares; SISMANA: Sistema de Informação para o Controle do Câncer de Mama; UNACON: Unidades de Assistência de Alta Complexidade. 
rando-se os indicadores entre as regiões e os grupos etários (40-49, 50-59, 60-69 e 70 anos e mais).

Para estimar a população feminina SUS dependente, em cada região e grupo etário, foi considerado o total de mulheres registradas pelo Censo de 2010 15, excluindo-se a proporção que informou ser portadora de plano de saúde privado na Pesquisa Nacional por Amostras de Domicílios 2008 (PNAD-2008) 16. Foram adotados os seguintes percentuais de população com plano de saúde privado: $13,3 \%$ para a Região Norte; $13,2 \%$ para a Região Nordeste; $35,6 \%$ para a Região Sudeste; $30 \%$ para a Região Sul; e 24,6\% para a Região Centro-Oeste.

\section{- Mortalidade por câncer de mama}

Para a construção de taxas de mortalidade, os óbitos com código C50 na Classificação Internacional de Doenças, 10a revisão (CID-10), foram extraídos do SIM, e os denominadores populacionais foram os do censo populacional de 2010 (IBGE. http:// www.ibge.gov.br). Para todo o país e regiões, foram calculadas taxas de mortalidade brutas e padronizadas por idade pela população do Brasil e taxas específicas por grupos de idade (40-49, 50-59, 60-69 e 70 anos e mais).

\section{- Razão de mamografias por mulheres segundo faixa etária}

Para estimar a cobertura da mamografia, utilizou-se uma razão tendo, no numerador, o número de exames mamográficos realizados no SUS e, no denominador, a metade do número de mulheres SUS dependentes, dado que a recomendação para o rastreamento é bienal. Essa razão foi calculada para cada uma das quatro faixas etárias estudadas (40-49, 50-59, 60-69 e 70 e mais anos) para o Brasil e regiões.

Ao comparar os dados registrados no SIA e no SISMAMA, em 2010, constatou-se, respectivamente, 3.126 .283 e 2.474 .413 mamografias em mulheres com 40 anos e mais, o que implica que $20,7 \%$ dos procedimentos só foram registrados no SIA. Assim, para o cálculo do indicador de cobertura, optou-se por considerar o maior valor, tomando-se, por base, os dados do SIA, independente da indicação clínica.

No SISMAMA, as mamografias com indicação de rastreamento ou de diagnóstico são registradas como um exame por mulher.

No SIA, são registrados os procedimentos conforme a indicação no pedido (i.e. rastreamento ou diagnóstico). Quando o pedido é para rastreamento, o registro de mamografia bilateral aparece como um único procedimento por mulher. Se o pedido é para diagnóstico, ou quando esse exame é feito para paciente já tratada por câncer de mama, o exame é registrado como referente a dois procedimentos de mamografia unilateral. Por esse motivo, no cálculo do indicador da razão de cobertura, quando a indicação no SIA apareceu como sendo de diagnóstico, utilizou-se metade dos procedimentos de mamografia unilateral de forma a equivaler ao número de mulheres examinadas.

\section{- Seguimento de lesões suspeitas na mamografia}

O Ministério da Saúde recomenda que todas as mulheres com BI-RADS 4 ou 5 devem ser submetidas à biópsia. Como nas bases do SIA não são informados os resultados dos laudos, a distribuição das mamografias, segundo categorias BI-RADS, foi feita a partir das informações do SISMAMA, e foram assumidas as mesmas proporções para o total informado no SIA

O número de biópsias realizadas foi acessado no SIA a partir do código de procedimento 0203020065 da Tabela de Procedimentos, Medicamentos, Órteses, Próteses e Materiais Especiais do SUS 17. A razão entre o número de biópsias efetuadas e o número de mamografias classificadas como BI-RADS 4-5 foi calculada, por região e grupo etário, como um indicador do grau de adequação do seguimento de achados mamográficos suspeitos de serem malignos.

\section{- Volume de cirurgias e sua adequação}

O número de cirurgias foi extraído do SIH, considerando todos os códigos referentes aos procedimentos cirúrgicos registrados com diagnósticos de neoplasia maligna invasiva da mama: 0410010111 - setorectomia/quadrantectomia; 0410010120 - setorectomia/quadrantectomia com esvaziamento ganglionar; 0416120024 - mastectomia radical com linfadenectomia axilar em oncologia; 0416120032 - mastectomia simples em oncologia; 0416120059 - segmentectomia de mama em oncologia; 0410010057 - mastectomia radical com linfadenectomia e 0410010065 - mastectomia simples 11. Taxas de cirurgia foram calculadas por grupo etário, para o Brasil e macrorregiões, tomando, como denominadores, o total de mulheres no mesmo grupo etário correspondente à população SUS dependente. A adequação do número de cirurgias foi investigada pelo cálculo das seguintes razões (por grupo etário e para o Brasil e macrorregiões): número de cirurgias/número de casos detectáveis e número de cirurgias/número estimado de casos novos de câncer da mama (ver seções Estimativa de Casos Novos de Câncer de Mama e Estimativa de Casos Detectáveis pelo Rastreamento Mamográfico). 


\section{- Estimativa de casos novos de câncer de mama}

Como não existe no Brasil um sistema nacional de registro de câncer de base populacional, a estimativa de número de casos novos de câncer de mama para o ano de 2010 para a população SUS dependente, por região e grupo etário, foi feita a partir do número de óbitos, aplicando-se a razão incidência/mortalidade. Optou-se por utilizar uma razão média de incidência/mortalidade de 4,35 , a qual foi estimada com base nos dados de Registros de Câncer de Base Populacional (RCBP), localizados em cidades brasileiras, apresentados em publicação recente do Instituto Nacional de Câncer José Alencar Gomes da Silva 18. Taxas de incidência foram estimadas por grupo etário, para o Brasil e macrorregiões, usando, como denominadores populacionais, a população SUS dependente.

\section{- Estimativa de casos detectáveis pelo rastreamento mamográfico}

Foi calculado o número de casos de câncer de mama detectáveis pela mamografia como sendo igual à soma de $20 \%$ dos resultados BI-RADS 4 com $80 \%$ dos BI-RADS 5 entre o total de mamografias realizadas (com indicação diagnóstica ou rastreamento), a partir de aproximação dos valores preditivos positivos dos resultados classificados como BI-RADS 4 e 5 apresentados em estudos publicados 19. As mesmas proporções de categorias BI-RADS no SISMAMA foram assumidas para o total de mamografias informado pelo SIA, e as taxas de casos detectáveis foram calculadas para a população SUS dependente por grupo etário, para o Brasil e macrorregiões.

\section{Resultados}

Comparando-se as taxas padronizadas por idade, constata-se que a mortalidade por câncer de mama é maior nas regiões Sul e Sudeste do país, com valores $40 \%$ superiores aos observados na Região Nordeste e duas vezes maiores que aqueles observados na Região Norte (Figura 1).

Em 2010, em todo o Brasil, foram realizadas, pelo SUS, 3.126.283 mamografias (com indicação diagnóstica ou de rastreamento) em mulheres a partir dos 40 anos, o que corresponde a 12,4\% das mulheres nessa faixa de idade. Nas faixas etárias alvo do rastreamento, a cobertura para a população SUS dependente, estimada a partir da razão exames/população alvo, foi de $32,2 \%$ para as mulheres de $50-59$ anos e de $25 \%$ para as de 60-69 anos. A mais baixa cobertura entre as mulheres de 60-69 anos foi observada na Região
Norte $(8,3 \%)$, e a mais elevada cobertura entre as mulheres de 50-59 anos foi observada na Região Sul (45,6\%) (Figura 2).

A cobertura nacional de mamografia para a faixa etária de 40-49, não preconizada para o rastreamento mamográfico, foi ligeiramente superior à observada na população alvo do programa de $60-69$ anos (26,5\% e $25 \%$, respectivamente), variando entre 9,5\%, na Região Norte, a 39,4\%, na Região Sul. Em contraste, a cobertura para as mulheres com 70 anos e mais foi apenas de 10,1\% (variando de 3,5\%, na Região Norte, a 14,4\%, na Região Sul).

A distribuição por categorias BI-RADS, segundo indicação da mamografia e de acordo com o SISMAMA, encontra-se na Tabela 2 . Apenas $4 \%$ de todas as mamografias realizadas entre mulheres de 40 anos e mais foram registradas nesse sistema como sendo de diagnóstico. Observa-se que, apesar de as proporções de categorias BI-RADS 4 e 5 serem maiores entre os exames mamográficos registrados como de diagnóstico, mais de $30 \%$ e mais de $40 \%$ das mamografias com essa indicação entre mulheres de 50-59 e 40-49 anos, respectivamente, aparecem com BI-RADS 1, indicando que parte das mamografias classificadas como de diagnóstico deve ter sido de rastreamento. Da mesma forma, erros no registro da indicação de rastreamento podem ter acontecido para mulheres nos grupos etários de 40-49 e de 70 anos e mais, nos quais apenas $4-5 \%$ dos exames foram registrados como de diagnóstico (porcentagem similar à observada para a população alvo do rastreamento).

A relação entre o número de biópsias e o número de mamografias com indicação para a realização desse exame (resultados de mamografias BI-RADS 4 e 5) se mostra bem desfavorável (0,36 para o Brasil nas mulheres de 40 anos e mais), sendo a pior relação encontrada na Região Sul para mulheres de todas as faixas etárias. Essa razão foi melhor no Nordeste e na faixa etária de 60 a 69 anos em todas as regiões (Tabela 3).

No SUS, foram realizadas 17.598 cirurgias no Brasil, em 2010, para o câncer de mama invasivo em mulheres a partir dos 40 anos de idade. Dessas, $32 \%$ foram para mulheres entre $40-49$ anos, $30 \%$ entre $50-59$ anos, $21 \%$ entre $60-69$ anos e $17 \%$ para aquelas com 70 anos e mais. Houve predomínio nessas três faixas etárias das cirurgias classificadas como segmentectomias, setorectomias ou quadrantectomias com esvaziamento axilar. Contudo, a porcentagem de mastectomias cresceu com o aumento da faixa etária (Tabela 4).

A relação entre o número de cirurgias e o número de casos detectáveis pelo rastreamento para todo o país foi de 1,38, 1,15, 1,35 e 1,84 para as faixas etárias de 40-49, 50-59 anos, 60-69 anos e 70 e mais, respectivamente. As razões observadas 
Figura 1

Mortalidade * por câncer de mama feminina, taxas brutas e padronizadas **. Brasil e regiões, 2010.

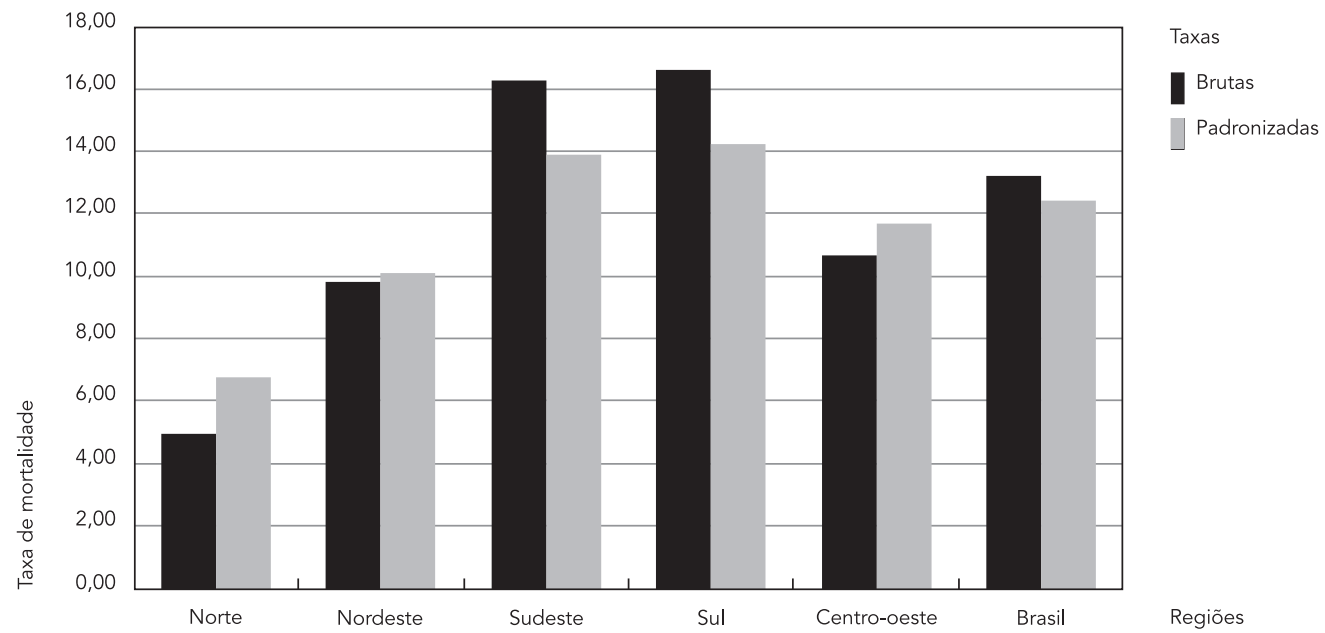

Fonte: Sistema de Informações sobre Mortalidade (Departamento de Informática do SUS. http://www2.datasus.gov.br/ DATASUS/index.php?area=02); Censo Demográfico 2010 (Instituto Brasileiro de Geografia e Estatística.

http://www.ibge.gov.br).

* Taxas por 100.000 mulheres;

** Padronizadas por idade pela população Brasil do Censo Demográfico 2010.

Figura 2

Cobertura estimada * de mamografia por faixa etária (anos). Brasil e regiões, 2010.

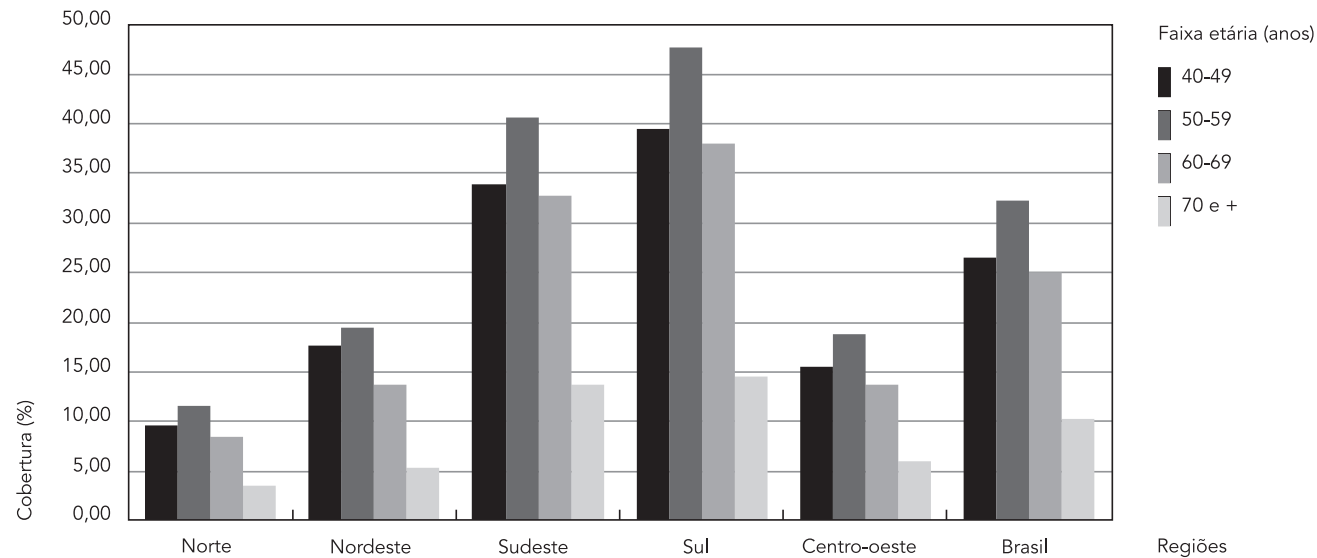

Fonte: Sistema de Informação do Controle do Câncer de Mama (SISMAMA); Sistema de Informações Ambulatoriais (SIA); (Departamento de Informática do SUS. http://www2.datasus.gov.br/DATASUS/index.php?area=02). Censo Demográfico 2010 (Instituto Brasileiro de Geografia e Estatística. http://www.ibge.gov.br).

* Estimada a partir da razão entre número de exames mamográficos informados no SIA em 2010 e a metade da população de mulheres no ano de 2010. 
Tabela 2

Distribuição de categorias de BI-RADS segundo indicação clínica das mamografias realizadas entre mulheres no SUS, por faixa etária, Brasil, 2010.

\begin{tabular}{|c|c|c|c|c|c|c|c|c|c|c|c|c|}
\hline \multirow[t]{3}{*}{ Categoria } & \multicolumn{12}{|c|}{ Faixa etária (anos) } \\
\hline & \multicolumn{3}{|c|}{$40-49$} & \multicolumn{3}{|c|}{$50-59$} & \multicolumn{3}{|c|}{$60-69$} & \multicolumn{3}{|c|}{70 e + } \\
\hline & $\mathrm{R}(\%)$ & D (\%) & $R+D(\%)$ & R (\%) & D (\%) & R+D (\%) & R (\%) & D (\%) & $R+D(\%)$ & R (\%) & D (\%) & $R+D(\%)$ \\
\hline \multicolumn{13}{|l|}{ BI-RADS } \\
\hline 0 & 12,1 & 13,5 & 12,2 & 11,3 & 12,2 & 11,3 & 10,0 & 10,3 & 10,0 & 9,5 & 10,4 & 9,5 \\
\hline 1 & 53,9 & 44,1 & 53,5 & 42,4 & 34,1 & 42 & 29,9 & 27,2 & 29,8 & 18,5 & 19,5 & 18,6 \\
\hline 2 & 30,7 & 29,5 & 30,6 & 42,3 & 37,1 & 42,1 & 55,6 & 44,6 & 55,1 & 66,6 & 51 & 65,8 \\
\hline 3 & 2,1 & 7,4 & 2,3 & 2,7 & 9,7 & 2,9 & 2,9 & 9,7 & 3,2 & 3,0 & 7,9 & 3,3 \\
\hline 4 & 1,1 & 3,7 & 1,2 & 1,2 & 4,4 & 1,4 & 1,4 & 5,1 & 1,5 & 1,9 & 5,7 & 2,1 \\
\hline 5 & 0,1 & 0,3 & 0,1 & 0,1 & 0,5 & 0,2 & 0,2 & 0,8 & 0,3 & 0,5 & 1,7 & 0,5 \\
\hline \multirow[t]{2}{*}{6} & & 1,4 & 0,1 & & 1,9 & 0,1 & & 2,2 & 0,1 & & 3,9 & 0,2 \\
\hline & 100,0 & 100,0 & 100,0 & 100,0 & 100,0 & 100,0 & 100,0 & 100,0 & 100,0 & 100,0 & 100,0 & 100,0 \\
\hline Total * & 944.166 & 41.404 & 985.570 & 863.547 & 34.546 & 898.093 & 423.543 & 17.340 & 440.883 & 149.622 & 7.916 & 157.538 \\
\hline$\%$ & 95,8 & 4,2 & 100,0 & 96,2 & 3,8 & 100,0 & 96,1 & 3,9 & 100,0 & 95,0 & 5,0 & 100,0 \\
\hline
\end{tabular}

Fonte: Sistema de Informação do Controle do Câncer de Mama (SISMAMA).

BI-RADS: Breast Imaging Reporting and Data System; D: diagnóstica; R: rastreamento.

* Esses dados excluem cerca de $21 \%$ de mamografias registradas apenas no SIA e para os quais não existe informação sobre a categoria BI-RADS.

Tabela 3

Número de biópsias e número de mamografias com resultados classificados com categorias BI-RADS 4 e 5 entre mulheres

SUS dependentes, por faixa etária, Brasil e regiões, 2010.

\begin{tabular}{|c|c|c|c|c|c|c|}
\hline Grupo etário (anos) & Norte & Nordeste & Sudeste & Sul & Centro-oeste & Brasil \\
\hline \multicolumn{7}{|l|}{$40-49$} \\
\hline Número de biópsias (B) & 200 & 1.812 & 2.028 & 370 & 291 & 4.701 \\
\hline Número de BI-RADS 4-5 * & 377 & 3115 & 7.643 & 4.415 & 3.627 & 16.243 \\
\hline Razão B/BI-RADS 4-5 & 0,53 & 0,58 & 0,27 & 0,08 & 0,08 & 0,29 \\
\hline \multicolumn{7}{|l|}{$50-59$} \\
\hline Número de biópsias (B) & 109 & 1.075 & 2.697 & 516 & 320 & 4.717 \\
\hline Número de BI-RADS 4-5 * & 349 & 2.755 & 8.953 & 4.480 & 761 & 17.259 \\
\hline Razão de B/BI-RADS 4-5 & 0,31 & 0,39 & 0,30 & 0,12 & 0,42 & 0,27 \\
\hline \multicolumn{7}{|l|}{$60-69$} \\
\hline Número de biópsias & 86 & 1.048 & 3.970 & 680 & 313 & 6.097 \\
\hline Número de BIRADS $4-5$ * & 160 & 1.466 & 5.158 & 2.542 & 375 & 9.679 \\
\hline Razão B/BI-RADS 4-5 & 0,54 & 0,71 & 0,77 & 0,27 & 0,84 & 0,63 \\
\hline \multicolumn{7}{|l|}{70 e mais } \\
\hline Número de biópsias (B) & 11 & 280 & 1.241 & 201 & 58 & 1.791 \\
\hline Número de BIRADS $4-5$ * & 76 & 786 & 3.087 & 1.011 & 145 & 5.119 \\
\hline Razão B/BI-RADS 4-5 & 0,14 & 0,36 & 0,40 & 0,20 & 0,40 & 0,35 \\
\hline \multicolumn{7}{|l|}{ Total } \\
\hline Número de biópsias (B) & 406 & 4.215 & 9.936 & 1.767 & 982 & 17.306 \\
\hline Número de BI-RADS 4-5 * & 962 & 8.124 & 24.860 & 12.446 & 4.865 & 48.323 \\
\hline Razão B/BI-RADS 4-5 & 0,42 & 0,52 & 0,40 & 0,14 & 0,20 & 0,36 \\
\hline
\end{tabular}

Fonte: Sistema de Informações Ambulatoriais (SIA) e Sistema de Informações Hospitalares (SIH); (Departamento de Informática do SUS. http://www2.datasus.gov.br/DATASUS/index.php?area=02).

BI-RADS: Breast Imaging Reporting and Data System.

* Usando as mesmas proporções informadas pelo Sistema de Informação do Controle do Câncer de Mama no total de mamografias informado no SIA. 
Tabela 4

Distribuição de procedimentos cirúrgicos para o câncer de mama realizados pelo SUS, por faixa etária, Brasil, 2010.

\begin{tabular}{|c|c|c|c|c|c|c|c|c|}
\hline \multirow[t]{3}{*}{ Procedimentos cirúrgicos } & \multicolumn{8}{|c|}{ Grupo etário (anos) } \\
\hline & \multicolumn{2}{|c|}{$40-49$} & \multicolumn{2}{|c|}{$50-59$} & \multicolumn{2}{|c|}{$60-69$} & \multicolumn{2}{|c|}{$70+$} \\
\hline & $\mathbf{n}$ & $\%$ & $\mathrm{n}$ & $\%$ & $\mathrm{n}$ & $\%$ & $\mathrm{n}$ & $\%$ \\
\hline Mastectomia & 409 & 7,34 & 441 & 8,45 & 288 & 7,68 & 289 & 9,46 \\
\hline Mastectomia com esvaziamento & 2.571 & 46,16 & 2.507 & 48,01 & 1.984 & 52,91 & 1.785 & 58,41 \\
\hline Setorectomia/Segmentectomia/Quadrantectomia & 1.707 & 30,65 & 1.402 & 26,85 & 839 & 22,37 & 566 & 18,52 \\
\hline Setorectomia/Segmentectomia/Quadrantectomia com esvaziamento & 883 & 15,85 & 872 & 16,70 & 639 & 17,04 & 416 & 13,61 \\
\hline Total & 5.570 & 100,00 & 5.222 & 100,00 & 3.750 & 100,00 & 3.056 & 100,00 \\
\hline
\end{tabular}

Fonte: Sistema de Informações Hospitalares (SIH); (Departamento de Informática do SUS. http://www2.datasus.gov.br/DATASUS/index.php?area=02).

Tabela 5

Incidência, detecção e cirurgias: números absolutos, taxas e razões por faixa etária entre mulheres SUS dependentes, Brasil e regiões, 2010.

\begin{tabular}{|c|c|c|c|c|c|c|c|c|c|c|c|c|}
\hline \multirow[t]{2}{*}{ Faixa etária (anos) } & \multicolumn{2}{|c|}{ Norte } & \multicolumn{2}{|c|}{ Nordeste } & \multicolumn{2}{|c|}{ Sudeste } & \multicolumn{2}{|c|}{ Sul } & \multicolumn{2}{|c|}{ Centro-oeste } & \multicolumn{2}{|c|}{ Brasil } \\
\hline & $\mathrm{n}$ & Taxa * & $\mathrm{n}$ & Taxa * & $\mathrm{n}$ & Taxa * & $\mathrm{n}$ & Taxa * & $\mathrm{n}$ & Taxa * & $\mathrm{n}$ & Taxa * \\
\hline \multicolumn{13}{|l|}{$40-49$} \\
\hline Incidência (I) ** & 347 & 48,72 & 1.929 & 67,96 & 3.079 & 82,75 & 1.127 & 80,14 & 479 & 66,70 & 6.961 & 74,08 \\
\hline 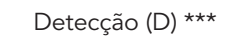 & 104 & 14,57 & 804 & 28,30 & 1.877 & 50,45 & 1.046 & 74,40 & 197 & 27,40 & 4.027 & 42,86 \\
\hline Cirurgias (C) & 199 & 27,94 & 1.367 & 48,15 & 2.429 & 65,28 & 1.149 & 81,73 & 426 & 59,40 & 5.570 & 59,28 \\
\hline Razão C/D & & 1,92 & & 1,70 & & 1,29 & & 1,10 & & 2,17 & & 1,38 \\
\hline Razão C/I & & 0,57 & & 0,71 & & 0,79 & & 1,02 & & 0,89 & & 0,80 \\
\hline Razão D/I & & 0,30 & & 0,42 & & 0,61 & & 0,93 & & 0,41 & & 0,58 \\
\hline \multicolumn{13}{|l|}{$50-59$} \\
\hline Incidência (I) ** & 392 & 83,73 & 2.605 & 128,70 & 4.732 & 160,20 & 1.666 & 151,10 & 656 & 135,00 & 10.051 & 142,90 \\
\hline 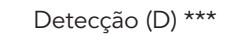 & 101 & 21,65 & 780 & 38,55 & 2.343 & 79,34 & 1.136 & 103,10 & 193 & 39,70 & 4.555 & 64,74 \\
\hline Cirurgias (C) & 182 & 38,85 & 1.043 & 51,53 & 2.569 & 86,98 & 1.059 & 96,05 & 369 & 75,80 & 5.222 & 74,22 \\
\hline Razão C/D & & 1,79 & & 1,34 & & 1,10 & & 0,93 & & 1,91 & & 1,15 \\
\hline Razão C/I & & 0,46 & & 0,40 & & 0,54 & & 0,64 & & 0,56 & & 0,52 \\
\hline Razão D/I & & 0,26 & & 0,30 & & 0,50 & & 0,68 & & 0,29 & & 0,45 \\
\hline \multicolumn{13}{|l|}{$60-69$} \\
\hline Incidência (I) ** & 245 & 91,78 & 1.907 & 139,90 & 3.916 & 213,60 & 1.702 & 245,90 & 531 & 190,00 & 8.302 & 187,20 \\
\hline 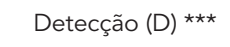 & 51 & 19,02 & 459 & 33,67 & 1.455 & 79,33 & 696 & 100,60 & 113 & 40,40 & 2.774 & 62,54 \\
\hline Cirurgias (C) & 121 & 45,30 & 775 & 56,87 & 1.908 & 104,10 & 762 & 110,10 & 184 & 65,80 & 3.750 & 84,55 \\
\hline Razão C/D & & 2,38 & & 1,69 & & 1,31 & & 1,09 & & 1,63 & & 1,35 \\
\hline Razão C/I & & 0,49 & & 0,41 & & 0,49 & & 0,45 & & 0,35 & & 0,45 \\
\hline Razão D/l & & 0,21 & & 0,24 & & 0,37 & & 0,41 & & 0,21 & & 0,33 \\
\hline
\end{tabular}

(continua) 
Tabela 5 (continuação)

\begin{tabular}{|c|c|c|c|c|c|c|c|c|c|c|c|c|}
\hline \multirow[t]{2}{*}{ Faixa etária (anos) } & \multicolumn{2}{|c|}{ Norte } & \multicolumn{2}{|c|}{ Nordeste } & \multicolumn{2}{|c|}{ Sudeste } & \multicolumn{2}{|c|}{ Sul } & \multicolumn{2}{|c|}{ Centro-Oeste } & \multicolumn{2}{|c|}{ Brasil } \\
\hline & $\mathrm{n}$ & Taxa * & $\mathrm{n}$ & Taxa * & $\mathrm{n}$ & Taxa * & $\mathrm{n}$ & Taxa * & $\mathrm{n}$ & Taxa * & $\mathrm{n}$ & Taxa * \\
\hline \multicolumn{13}{|l|}{70 e mais } \\
\hline Incidência (I) ** & 283 & 137,80 & 2.745 & 218,80 & 5.858 & 356,00 & 2.153 & 366,60 & 597 & 289,00 & 11.635 & 298,40 \\
\hline Detecção (D) *** & 32 & 15,70 & 269 & 21,47 & 999 & 60,70 & 315 & 53,57 & 49 & 23,90 & 1.664 & 42,69 \\
\hline Cirurgias (C) & 64 & 31,19 & 589 & 46,96 & 1.709 & 103,90 & 560 & 95,36 & 134 & 64,90 & 3.056 & 78,38 \\
\hline Razão C/D & & 1,99 & & 2,19 & & 1,71 & & 1,78 & & 2,71 & & 1,84 \\
\hline Razão C/I & & 0,23 & & 0,21 & & 0,29 & & 0,26 & & 0,22 & & 0,26 \\
\hline Razão D/l & & 0,11 & & 0,10 & & 0,17 & & 0,15 & & 0,08 & & 0,14 \\
\hline
\end{tabular}

Fonte: Sistema de Informação do Controle do Câncer de Mama (SISMAMA); Sistema de Informaç̃̃es Ambulatoriais (SIA); Sistema de Informações Hospitalares (SIH) (Departamento de Informática do SUS. http://www2.datasus.gov.br/DATASUS/index.php?area=02); Censo Demográfico 2010 (Instituto Brasileiro de Geografia e Estatística. http://www.ibge.gov.br).

* Taxas brutas por 100.000 mulheres calculadas para a população SUS dependente;

** Número de casos novos de câncer de mama estimado considerando-se uma razão incidência/mortalidade de 4,35. Taxas calculadas para a população SUS dependente;

*** Número de casos de câncer de mama invasivo detectáveis por mamografia estimado como correspondendo a: 0,2 dos resultados BI-RADS 4 + 0,8 dos resultados BI-RADS 5, conforme informado no SISMAMA e assumindo as mesmas proporções para o total informado no SIA. Taxas calculadas para a população SUS dependente.

foram sempre superiores a um, com exceção do encontrado na Região Sul entre 50 e 59 anos $(0,93)$. Na Região Norte, as razões cirurgias/casos detectáveis pelo rastreamento variaram entre 1,79 para mulheres de 50-59 anos a 2,38 para as com 60-69 anos. Por outro lado, na Região Centro-oeste, a razão entre cirurgias e casos detectáveis foi de 2,17 para as de 40-49 anos e de 2,71 para aquelas com 70 anos e mais. No entanto, ao se comparar o número de cirurgias com o número de casos incidentes estimados, as razões são inferiores a um, com exceção do observado entre as mulheres de 40-49 anos na Região Sul $(1,02)$. Observa-se, ainda, que a razão entre casos detectáveis pelo rastreamento e casos incidentes estimados mostra uma relação muito desfavorável nas faixas etárias alvo do rastreamento (0,45 entre 50-59 anos e 0,33 entre 60-69 anos), sendo inferior ao observado para mulheres entre 40 a 49 anos $(0,58)$. O valor mais elevado para essa razão foi observado entre as mulheres dessa faixa mais jovem, na Região Centro-oeste $(0,93)$. Mais uma vez as regiões Norte e Nordeste aparecem com os piores indicadores e justamente nas faixas etárias recomendadas para o rastreamento cujas razões foram iguais ou menores que $30 \%$. (Tabela 5).

\section{Discussão}

No Brasil, como observado em outros países 2, há um claro gradiente na mortalidade por câncer de mama que varia com o grau de desenvolvimento socioeconômico. Observa-se maior ocorrência nas regiões Sudeste e Sul, as mais abastadas do país; as regiões Norte, Nordeste e Centro-oeste, que, além de menos desenvolvidas, têm população mais jovem, apresentam taxas menores mesmo após a padronização por idade.

As medidas de controle incluem a detecção e o tratamento precoce pelo aumento de alerta para o câncer de mama, o rastreamento organizado e a melhora dos procedimentos de diagnóstico e tratamento, as quais devem ser implementadas dentro de critérios rigorosos de avaliação de seu impacto em nível populacional.

A razão entre mamografias e população alvo do rastreamento, uma medida indireta de cobertura, mostrou-se baixa em todas as faixas etárias analisadas. É possível que esses valores representem uma superestimação da verdadeira cobertura uma vez que: (i), no seu cálculo, foram incluídas não só mamografias de rastreio como também de diagnóstico; e (ii) não foi possível tomar, em consideração, o número de repetições de mamografias que uma mulher pode ter realizado durante 2010. Esses valores foram inferiores aos observados em países em que foram organizados programas de rastreio de base populacional, como, por 
exemplo, na Inglaterra, onde a cobertura foi de $73 \%$ em 2010/2011 20, e se aproximam daqueles relatados em cidades latino-americanas, incluindo São Paulo 21.

O número de biópsias comparado ao número de casos que demandariam seguimento para esclarecimento diagnóstico (resultados BI-RADS 4 e 5) mostra que apenas $27 \%$ das mulheres entre 50 e 59 anos com mamografias com BI-RADS 4 e 5 estão realizando biópsia, já na faixa etária entre aquelas com 60-69 anos, esse percentual sobe para $63 \%$. Foi na Região Sul onde essa relação se mostrou mais desfavorável (12\% e $27 \%$, respectivamente). Afastada a possibilidade de erros de registro, podem ser sugeridas algumas interpretações, como, por exemplo, que esteja ocorrendo o encaminhamento de casos para cirurgia sem biópsia realizada no SUS. Ao mesmo tempo, levandose em conta, o fato de que a razão entre cirurgias e casos estimados é maior do que a razão entre número de casos detectáveis e casos estimados, é possível que parte das cirurgias com diagnóstico de neoplasia maligna de mama seja, de fato, casos de doença benigna.

A escassez de biópsias correspondente à necessidade diagnóstica estimada pelo número de mamografias realizadas no país indica que o sistema de saúde ainda não está preparado para atender à demanda de mulheres que deveriam ser alvo das ações específicas de rastreamento e diagnóstico precoce para o câncer de mama.

Por outro lado, $46 \%$ do total de mamografias realizadas em 2010 foram feitas em faixas etárias não recomendadas para rastreamento pelo Ministério da Saúde 10. Sabe-se que a indicação médica é um forte preditor da realização da mamografia 22,23 , e que as sociedades científicas têm tido importante papel na difusão de recomendações distintas das preconizadas oficialmente, em especial no que diz respeito à idade. Também há divergências quanto à periodicidade, não sendo possível afastar a ideia de que os exames estejam sendo feitos fora do padrão bienal.

Embora o número de cirurgias registrado seja insuficiente para o número de casos estimados, é provável que uma proporção significativa de cirurgias esteja sendo realizada em mulheres com doença avançada, especialmente na Região Norte, onde foram vistas as mais altas razões cirurgias/ casos detectáveis. Além disso, não se pode afastar a possibilidade de estar havendo um excesso de cirurgias entre mulheres fora das faixas de rastreamento, o que precisa ser melhor investigado, dado que o rastreamento mamográfico antes dos 50 anos aumentaria o risco de sobrediagnóstico e falsos positivos, causando mais danos do que benefícios 24 . Com base nesse entendimento, a Força Tarefa de Serviços Preventivos nos Estados Unidos da América, em 2009, recomendou o rastreamento mamográfico a partir dos 50 anos 25 , o que já era seguido em vários países 26 .

O acesso ao diagnóstico e ao tratamento de câncer no Brasil é marcado pelas imensas desigualdades de oferta de assistência especializada. Há uma grande concentração de serviços credenciados no SUS de quimioterapia e radioterapia nas regiões Sudeste e Sul e uma ausência, quase total, na Região Norte 27 , o que certamente afeta o prognóstico de mulheres acometidas pela doença fora dos grandes centros urbanos do país. Estudo que analisou o fluxo de pacientes com câncer de mama tratadas no SUS mostra que os serviços especializados de cirurgia, radioterapia e quimioterapia são concentrados nas cidades grandes, e que uma proporção considerável de pacientes mora a mais de $150 \mathrm{~km}$ desses serviços 28 . Todavia, mesmo no Estado do Rio de Janeiro, um dos estados de melhor nível socioeconômico, evidenciou-se que $44 \%$ dos casos de câncer de mama foram diagnosticados em fase avançada, embora $68 \%$ dessas mulheres residissem em municípios com serviço oncológico credenciado 29.

Apesar das baixas coberturas de mamografia entre as mulheres que dependem do SUS, é inegável que vem havendo expansão de oferta desse exame no país. Estudo com dados das PNAD de 2003 e 2008 concluiu que houve aumento na realização de mamografia em todas as faixas etárias, mesmo naquelas em que o rastreamento não é recomendado. Esse aumento foi mais expressivo $(42 \%)$ entre as mulheres sem planos de saúde, para as quais a prevalência passou de $33 \%$ para $47 \%$. Para as mulheres na faixa etária preconizada pelo Ministério da Saúde (50-69 anos), também se observou aumento de $30 \%$, que se relacionou positivamente com aumento de renda familiar e escolaridade, e para as que possuem planos de saúde ${ }^{30}$. É marcante a observação de que mulheres residentes em áreas metropolitanas apresentam uma chance três vezes maior de acesso à mamografia do que as residentes em outras áreas.

Comparando-se a discrepância entre as coberturas calculadas a partir de exames realizados pela população SUS dependente e a informação obtida na PNAD que incluiu toda a população, fica evidente que o acesso à mamografia é maior para quem dispõe de assistência de saúde suplementar. A parcela de mulheres que informam ter realizado mamografia em serviços privados foi maior do que a das que disseram tê-lo feito em serviços próprios ou contratados do SUS, tanto em 2003 (64,7\% x $42,5 \%)$ quanto em 2008 (72,7\% x 47\%) 30.

Os resultados apontados no presente estudo levantam a possibilidade de que esteja ocorrendo uma relação muito desfavorável entre o que seria detectável a partir dos exames alterados de 
mamografia e o número de casos estimados para todas as regiões do Brasil. Mais de 50\% dos casos estimados de câncer de mama entre mulheres de 50-59 anos no país que não possuem assistência de saúde suplementar não estariam sendo detectados por mamografia; entre 60-69 anos, o número de casos não detectáveis chega a $67 \%$. E foram nas regiões mais pobres do país, Norte e Nordeste, que se evidenciaram as piores razões entre os casos suspeitos pela mamografia e o número de casos estimados.

As informações de inquéritos domiciliares dizem respeito à população como um todo (incluindo aquelas que possuem planos privados e não usam os serviços do SUS), são, porém, aferidas a partir de autorrelato, podendo haver sub ou superestimação das coberturas de ações de rastreamento. Ao passo que os dados registrados nos sistemas de informações do SUS se referem a pessoas que foram atendidas na rede própria ou de serviços contratados do SUS, não incluem assim aqueles sem acesso a esses serviços.

Entre as limitações deste estudo, deve ser destacado que seus resultados tiveram, como fonte, sistemas de informações com diferentes graus de cobertura e variações regionais na completude e qualidade da informação ${ }^{31}$. Em relação ao SIM, há, em geral, um consenso de que os óbitos por neoplasias são mais bem registrados, ainda que parte deles possa estar incluída entre as causas mal definidas que vêm diminuindo marcadamente no país 32 . O diagnóstico e o tratamento do câncer de mama implicam assistência hospitalar, e as informações costumam ser mais fidedignas relativamente a outras causas. O SIA e o SIH, embora criados com finalidade contábil e gerencial, constituem-se em fontes de dados valiosas para a pesquisa sobre a assistência oncológica 33. Quanto ao SISMAMA, por ter sido implantado recentemente, sua qualidade foi pouco avaliada ${ }^{13}$. Seus limites foram apontados no presente artigo.

As análises contemplaram os totais de procedimentos (exames e cirurgias) realizados, não sendo possível seguir a trajetória de cada mulher a partir do SISMAMA, que, até o momento, registra o exame e não a mulher. Além disso, há perdas no total de informações da base de dados do SISMAMA, motivo pelo qual considerou-se o total de exames registrados no SIA. Espera-se que, com o aprimoramento desses sistemas, seja possível avaliar o seguimento dos casos rastreados e a adequação do tratamento, permitindo avaliar a adesão ao rastreamento e as barreiras de acesso aos serviços do SUS.

Outra limitação que deve ser considerada é que a estimativa do número de casos incidentes se baseou em uma razão única entre incidência/ mortalidade, ignorando diferenças de sobrevida entre os grupos etários e as regiões do país. Por exemplo, não considerou a possibilidade de que, nas áreas mais carentes socioeconomicamente, a sobrevida seria menor, o que diminuiria essa razão e, consequentemente, a estimativa de casos novos.

Pode-se, ainda, pensar que a cobertura da mamografia avaliada apenas com exames gerados na rede de serviço do SUS esteja subestimada caso algumas mulheres tenham realizado o exame em serviços privados. O aumento da cobertura de plano de saúde suplementar no país pode estar gerando algumas inconsistências verificadas no estudo; parte dos planos privados de saúde oferecem cobertura para exames complementares ou mesmo biópsias, mas, nem sempre cobrem procedimentos cirúrgicos mais complexos, levando as pessoas a recorrerem ao SUS para realizá-los.

O Brasil tem experimentado, nas últimas décadas, um intenso processo de urbanização e de mudanças no estilo de vida, especialmente para as mulheres, com progressivo envelhecimento populacional, consequente ao aumento da expectativa de vida ao nascer, redução marcada da fecundidade e aumento da obesidade e da incidência de doenças crônicas ${ }^{34}$. O controle de fatores de risco modificáveis associados a doenças crônicas não transmissíveis é contemplado dentro do Plano de Ações Estratégicas para o Enfrentamento das Doenças Crônicas não Transmissíveis no Brasil, 2011-2020 35. Entre esses fatores, o uso de álcool, o excesso de peso e a inatividade física após a menopausa se associam ao aumento de risco para o câncer de mama. No entanto, os principais fatores de risco, tais como idade avançada da primeira gestação, baixa paridade e amamentar por períodos curtos, são menos passíveis a intervenções de saúde pública, principalmente nas sociedades modernas em que há, cada vez mais, uma maior participação profissional e social das mulheres. Assim, o diagnóstico e tratamento precoces, para os quais o rastreamento é um componente essencial, são os meios mais efetivos para a redução da mortalidade por câncer de mama.

As taxas de mortalidade por câncer de mama, padronizadas por idade, de mulheres residentes nas capitais estão em declínio nas regiões Sul e Sudeste, mesmo antes da implementação das recomendações governamentais sobre rastreamento em 2004 36. Contudo, nas regiões Norte, Nordeste e Centro-oeste, a mortalidade continua crescente tanto nas capitais quanto nos municípios do interior, o que traduz as desigualdades de acesso aos serviços, que também ficaram evidenciadas no presente estudo.

Os resultados apresentados permitem uma primeira avaliação sobre a correspondência entre 
as diversas etapas do processo de rastreamento com base em dados secundários oficiais do SUS no Brasil e grandes regiões. Há grande defasagem em termos de cobertura de mamografia e biópsias em todo o país, especialmente nas regiões Norte e Nordeste. A existência de uma leve tendência a uma maior cobertura de mamografia para as mulheres de 50-59 anos se comparada às outras faixas etárias analisadas pode estar refletindo uma pequena adesão às recomendações de rastreamento do Ministério da Saúde. Por outro lado, o excesso de intervenções cirúrgicas entre as mais jovens pode sugerir a existência de sobrediagnóstico, merecendo uma investigação mais cuidadosa dos efeitos dessa possibilidade na saúde e na vida dessas mulheres. No entanto, esses achados foram feitos a partir de estimativas com parâmetros médios de bancos secundários, que ainda trazem problemas de qualidade e cobertura da informação, e podem não corresponder de forma precisa à realidade.

O objetivo final do rastreamento do câncer de mama é a redução da mortalidade. Como tal, é fundamental que as ações realizadas sejam avaliadas em função do seu impacto na mortalidade, o que não foi possível ser feito na presente análise. Contudo, a baixa cobertura mamográfica, o seguimento inadequado de lesões radiológicas suspeitas e o déficit de acesso a tratamento cirúrgico apontam para um baixo impacto das medidas de rastreamento na mortalidade por câncer de mama no Brasil. A melhora desses indicadores, bem como a criação de mecanismos que permitam a monitorização do custo-efetividade do rastreamento mamográfico e de seu impacto devem ser encaradas como prioridades da política de câncer para o país.

\section{Resumen}

La reciente reducción de la mortalidad por cáncer de mama en los países de altos ingresos se atribuye a la detección precoz y las mejoras en el tratamiento. El cáncer de mama es el cáncer femenino más común en Brasil y desde 2004 el gobierno recomienda el examen clínico anual para las mujeres después de los 40 años y la mamografía bienal entre 50 a 69 años. Este trabajo investiga el grado de aplicación de estas recomendaciones a partir de datos del Sistema de Información de Salud, en 2010 por grupos de edad. Los resultados mostraron una baja cobertura de la mamografía en la población objetivo (32\%: 50-59, 25\%: 60-69 años). La proporción de mujeres con hallazgos radiológicos anormales se sometió a biopsia también fue baja (27\%: 50-59, 63\%: 60-69 años). El número de cirugías para el cáncer de mama fue mayor que el número de casos detectados por la mamografía, pero mucho menos que el número estimado de casos nuevos para el año 2010. Existen marcadas diferencias regionales en el acceso a la detección temprana y la cirugía, siendo el acceso menor en la Región Norte y más alto en la Región Sur.

Neoplasias de la Mama; Detección Precoz del Cáncer; Salud de la Mujer; Tamizaje Masivo

\section{Colaboradores}

G. Azevedo e Silva foi responsável pela concepção do estudo e planejamento da análise, participou da extração e análise dos dados e de todas as etapas de redação do artigo, da revisão crítica do texto e aprovou a versão final. M. T. Bustamante-Teixeira contribuiu com a concepção do estudo, participou da extração e análise dos dados, da redação do artigo e aprovou a versão final. E. M. L. Aquino contribuiu com a redação do artigo e com a interpretação dos resultados, participou da revisão crítica do conteúdo intelectual e aprovou a versão final. J. G. Tomazelli contribuiu com a metodologia e com o levantamento dos dados, participou da análise dos dados e aprovou a versão final. I. dos-Santos-Silva contribui com a concepção do estudo, análise dos dados, interpretação dos resultados, revisão crítica do texto e aprovou a versão final. 


\section{Referências}

1. Porter PL. Global trends in breast cancer incidence and mortality. Salud Pública Mex 2009; 51 Suppl 2:S141-6.

2. Forouzanfar MH, Foreman KJ, Delossantos AM, Lozano R, Lopez AD, Murray CJ, et al. Breast and cervical cancer in 187 countries between 1980 and 2010: a systematic analysis. Lancet 2011; 378:1461-84.

3. Bray F, Jemal A, Grey N, Ferlay J, Forman D. Global cancer transitions according to the Human Development Index (2008-2030): a population-based study. Lancet Oncol 2012; 13:790-801.

4. Youlden DR, Cramb SM, Dunn NA, Muller JM, Pyke CM, Baade PD. The descriptive epidemiology of female breast cancer: an international comparison of screening, incidence, survival and mortality. Cancer Epidemiol 2012; 36:237-48.

5. Shapiro S. Evidence on screening for breast cancer from a randomized trial. Cancer 1977; 39:2772-82.

6. Miller AB, Howe GR, Wall C. The National Study of Breast Cancer Screening Protocol for a Canadian Randomized Controlled trial of screening for breast cancer in women. Clin Invest Med 1981; 4:227-58.

7. Shapiro S, Venet W, Strax P, Venet L, Roeser R. Selection, follow-up, and analysis in the Health Insurance Plan Study: a randomized trial with breast cancer screening. Natl Cancer Inst Monogr 1985; 67:65-74.

8. Independent UK Panel on Breast Cancer Screening. The benefits and harms of breast cancer screening: an independent review. Lancet 2012; 380:1778-86.

9. Cazap E, Buzaid AC, Garbino C, de la Garza J, Orlandi FJ, Schwartsmann G, et al. Breast cancer in Latin America: results of the Latin American and Caribbean Society of Medical Oncology/Breast Cancer Research Foundation expert survey. Cancer 2008; 113:2359-65.

10. Controle do câncer de mama-documento de consenso. Rev Bras Cancerol 2004; 50:77-90.

11. Ministério da Saúde. Portaria no $779 / 08$ de 31 de dezembro de 2008. Define o Sistema de Informação do Controle do Câncer de Mama (SISMAMA) como sistema oficial para o fornecimento dos dados informatizados dos procedimentos relacionados ao rastreamento e a confirmação diagnóstica do câncer de mama. Diário Oficial da União 2009; 2 jan.

12. Passman LJ, Farias AM, Tomazelli JG, de Abreu DM, Dias MB, de Assis M, et al. SISMAMA - implementation of an information system for breast cancer early detection programs in Brazil. Breast 2011; 20 Suppl 2:S35-9.

13. Santos SBL, Koch HA. Análise do Sistema de Informação do Programa de Controle do Câncer de Mama (SISMAMA) mediante avaliação de 1.000 exames nas cidades de Barra Mansa e Volta Redonda. Radiol Bras 2010; 43:295-301.

14. American College of Radiology. American College of Radiology BI-RADS ${ }^{\circ}$ ATLAS and MQSA: frequently asked questions (updated: DRAFT 11/29/12). Reston: American College of Radiology; 2012.
15. Travassos C, Martins M. Uma revisão sobre os conceitos de acesso e utilização de serviços de saúde. Cad Saúde Pública 2004; 20 Suppl 2:S190-8.

16. Instituto Brasileiro de Geografia e Estatística. Pesquisa Nacional por Amostra de Domicílios - um panorama da saúde no Brasil: acesso e utilização dos serviços, condições de saúde e fatores de risco e proteção à saúde (PNAD 2008). Rio de Janeiro: Instituto Brasileiro de Geografia e Estatística; 2010.

17. Ministério da Saúde. Portaria GM/MS no 2.848, de 6 de novembro de 2007. Publica a Tabela de Procedimentos, Medicamentos, Órteses, Próteses e Materiais Especiais - OPM do Sistema Único de Saúde. Diário Oficial da União 2007; 7 nov.

18. Instituto Nacional de Câncer José Alencar Gomes da Silva. Magnitude do câncer no Brasil: incidência, mortalidade e tendência. Rio de Janeiro: Instituto Nacional de Câncer José Alencar Gomes da Silva; 2012. (Informativo Vigilância do Câncer, 28).

19. Kestelman FP, Souza GA, Thuler LC, Martins G, Rolim de Freitas VA, Canella EO. Breast Imaging Reporting and Data System - BI-RADS: valor preditivo positivo das categorias 3, 4 e 5. Revisão sistemática da literatura. Radiol Bras 2007; 40:173-7.

20. The NHS Information Centre, Screening and Immunisations. Breast screening programme, England 2010-11. Leeds: NHS Information Centre for Health and Social Care Part of the Government Statistical Service; 2012.

21. Reyes-Ortiz CA, Freeman JL, Pelaez M, Markides KS, Goodwin JS. Mammography use among older women of seven Latin American and Caribbean cities. Prev Med 2006; 42:375-80.

22. Lima-Costa MF, Matos DL. Prevalência e fatores associados à realização da mamografia na faixa etária de 50-69 anos: um estudo baseado na Pesquisa Nacional por Amostra de Domicílios (2003). Cad Saúde Pública 2007; 23:1665-73.

23. Rauscher GH, Hawley ST, Earp JA. Baseline predictors of initiation vs. maintenance of regular mammography use among rural women. Prev Med $2005 ; 40: 822-30$.

24. The benefits and harms of breast cancer screening: an independent review. Lancet 2012; 380:1778-86.

25. U.S. Preventive Services Task Force. Screening for breast cancer: U.S. Preventive Services Task Force recommendation statement. Ann Intern Med 2009; 151:716-26, W-236.

26. Moss SM, Nystrom L, Jonsson H, Paci E, Lynge E, Njor S, et al. The impact of mammographic screening on breast cancer mortality in Europe: a review of trend studies. J Med Screen 2012; 19 Suppl 1:26-32.

27. Azevedo-e-Silva G, Bustamante-Teixeira MT, Guerra MR, Moura L. Tendências e controle do câncer e os 20 anos de Sistema Único de Saúde no Brasil. In: Departamento de Análise de Situação em Saúde, Secretaria de Vigilância em Saúde, Ministério da Saúde, organizador. Saúde Brasil 2008: 20 anos de SUS no Brasil. Brasília: Ministério da Saúde; 2009. p. 365-84. (Série G. Estatística e Informação em Saúde). 
28. Oliveira EXG, Melo ECP, Pinheiro RS, Noronha $\mathrm{CP}$, Carvalho MS. Acesso à assistência oncológica: mapeamento dos fluxos origem-destino das internações e dos atendimentos ambulatoriais. O caso do câncer de mama. Cad Saúde Pública 2011; 27: 317-26.

29. Brito C, Portela MC, Vasconcellos MTL. Assistência oncológica pelo SUS a mulheres com câncer de mama no Estado do Rio de Janeiro. Rev Saúde Pública 2005; 39:874-81.

30. Oliveira EX, Pinheiro RS, Melo EC, Carvalho MS. Condicionantes socioeconômicos e geográficos do acesso à mamografia no Brasil, 2003-2008. Ciênc Saúde Coletiva 2011; 16:3649-64.

31. Felix JD, Zandonade E, Amorim MHC, Castro DS. Avaliação da completude das variáveis epidemiológicas do Sistema de Informação sobre Mortalidade em mulheres com óbitos por câncer de mama na Região Sudeste: Brasil (1998 a 2007). Ciênc Saúde Coletiva 2012; 17:945-53.

32. Jorge MHPM, Gotlieb SLD, Laurenti R. O sistema de informações sobre mortalidade: problemas e propostas para o seu enfrentamento I - Mortes por causas naturais. Rev Bras Epidemiol 2002; 5: 197-211.
33. Brito C, Portela MC, Vasconcellos MTL. Avaliação da concordância de dados clínicos e demográficos entre Autorizações de Procedimento de Alta Complexidade Oncológica e prontuários de mulheres atendidas pelo Sistema Único de Saúde no Estado do Rio de Janeiro, Brasil. Cad Saúde Pública 2005; 21:1829-35.

34. Victora CG, Barreto ML, do Carmo Leal M, Monteiro CA, Schmidt MI, Paim J, et al. Health conditions and health-policy innovations in Brazil: the way forward. Lancet 2011; 377:2042-53.

35. Malta DC, Morais Neto OL, Silva Junior JB. Apresentação do plano de ações estratégicas para o enfrentamento das doenças crônicas não transmissíveis no Brasil, 2011 a 2022. Epidemiol Serv Saúde 2013; 20:425-38.

36. Azevedo-e-Silva G, Gamarra CJ, Girianelli VR, Valente JG. Tendência da mortalidade por câncer nas capitais e interior do Brasil entre 1980 e 2006. Rev Saúde Pública 2011; 45:1009-18.

Recebido em 30/Ago/2013

Versão final reapresentada em 12/Dez/2013

Aprovado em 09/Jan/2014 